\title{
AOR
}

Selected Papers of \#AolR2020:

The $21^{\text {st }}$ Annual Conference of the

Association of Internet Researchers

Virtual Event / 27-31 October 2020

\section{TRANSCODING BETWEEN HYPER-ANTAGONISTIC MILIEUS: STUDIES ON THE CROSS-PLATFORM RELATIONS BETWEEN RADICAL POLITICAL WEB SUBCULTURES}

\author{
Sal Hagen \\ University of Amsterdam \\ Emillie de Keulenaar \\ Simon Fraser University \\ Kings College London \\ Stijn Peeters \\ University of Amsterdam \\ Marc Tuters \\ University of Amsterdam \\ Tom Willaert \\ Vrije Universiteit Brussel \\ Jack Wilson \\ University of Warwick
}

This panel brings together research into the hyper-antagonistic politics and crossplatform relations of radical Web subcultures, focused primarily on the U.S. context in the mid to late 2010's. The papers share a concern with vernacular practices of "fringe" platforms favoured by an insurgent far-right movement and their relations to more "mainstream" social media. These fringe platforms engage in the production and propagation of vernacular artefacts often described as "memes". Derived from an earlier and arguably outdated paradigm in evolutionary biology, the meme metaphor is not particularly attuned to the relationality of the material "milieus" in which they are developed, adopted, and used. In an effort to describe these practices without falling

Hagen, S, de Keulenaar, E, Peeters, S, Tuters, M, Willaert, T, Wilson, J. (2020, October). Transcoding between Hyper-Antagonistic Milieus: Studies on the Cross-Platform Relations between Radical Political Web Subcultures. Panel presented at AoIR 2020: The 21 ${ }^{\text {th }}$ Annual Conference of the Association of Internet Researchers. Virtual Event: AolR. Retrieved from http://spir.aoir.org. 
back on biological or epidemiological metaphors, the papers in this panel engage with the concept of "transcoding between milieus" (Deleuze \& Guattari 1987, p. 322). Deleuze and Guattari describe milieus not as "unitary", but rather as coded by multiple "transversal" processes. According to this vitalist materialist philosophy, "not only does the living thing continually pass from one milieu to another, but the milieus pass into one another", such that "one milieu serves as the basis for another" (1987, p. 313). The papers in this panel thus describe a mutually shaping relationship between the material affordances and the vernacular practices of given fringe platforms, using "transcoding" to conceptualize how these practices in turn overlap across different "strata" of the Web. All of the papers furthermore share data-driven empirical approaches that use historical datasets from a variety of social media platforms in order to study patterns of shared political identity amongst online strangers engaged in perpetual antagonism - what we refer to as "hyper-antagonistic milieus". Such hyper-antagonism is best exemplified by the far-right activity emanating from the imageboard 4chan, which all the papers in this panel use datasets from, ranging from 2014 to the present.

The first paper conducts an empirical analysis on a cultural conflict between 4chan and Tumblr in 2014. It considers a series of cross-platform "raids" as a microcosm for elements that would later be provocatively framed as at the basis of an "online culture war". The second paper focuses on a different cross-platform relationship: between 4chan and YouTube. It does so through a case study of the "Kekistan" meme, an imaginary country of aggrieved denizens of the "deep vernacular Web" who jokingly consider themselves as being at war with "politically correct" liberal culture. In lieu of framing this relationship in terms of propaganda or quasi-biological metaphors, the paper theorizes a relationship of transcoding between the platforms in which 4chan's vernacular culture serves as the model. The penultimate paper looks at the crossplatform prevalence of specific vernacular terms originating on 4chan/pol/ as occurring in the comments and articles of Breitbart News, a media outlet whose significance has been connected with the Trump campaign's successful agenda setting in the 2016 election. In comparing these milieus from the period around the election, the paper interrogates whether the spread of /pol/'s vernacular may also indicate the spread of its hyper-antagonistic politics into a more mainstream space of political discussion. The final paper explores the cross-platform presence of fringe cultural artefacts after the "deplatforming" measures taken by platforms such as YouTube in 2019. In response to academic and journalistic reports about the effectiveness of deplatforming, it takes a temporal view before and after such measures in order to trace the migration of content to alternative platforms such as Bitchute, Gab, and Voat, as well their possible reappearance or "replatforming" on mainstream platforms. To this end, instead of following banned micro-celebrities, the paper studies the cross-platform robustness of vernacular, fringe, and hyper-antagonist discourse. Taken together, the panel offers a comprehensive assessment of a moment in time when participatory culture seemed to have taken a turn towards the reactionary; how it got there, how it reverberated across the Web, and how involved platforms have tried to respond.

\section{References}

Deleuze, G, Guattari, F. (1987). A Thousand Plateaus. Minneapolis: University of Minnesota Press. 


\title{
REMEMBERING THE 2014 TUMBLR-4CHAN RAIDS: A DATA-DRIVEN MICROHISTORY OF ONLINE POLITICIZATION THROUGH CROSS- SUBCULTURAL CONFLICT
}

\author{
Sal Hagen \\ University of Amsterdam
}

On 9 June 2014, a post on a Tumblr blog read: "join us on July 4th to celebrate our freedom and independence from racists by shutting down 4chan" (shutdown4chan, 2014). That Independence Day, several Tumblr users answered the call. Armed with loving messages and cute pictures of cats, the aim was to "detoxify" 4chan's infamous /b/ "Random" board, known for its nihilistic trolling subculture, shock value, and memes (Phillips, 2015). According to vernacular Web historians (Internet Historian, 2017), Tumblr's efforts were futile, merely invoking the collective retribution of 4chan's users: several Tumblr tags associated with gender and fandom were spammed with gore and hardcore porn. The "2014 4chan-Tumblr Raids", as the event came to be known, features a dramatic twist: not a Tumblr user, but someone from 4chan's/pol/ "Politically Incorrect" board, now infamous for its white supremacism, was allegedly behind the Tumblr post that kickstarted the controversy.

The utopian outlook that once framed "Twitter revolutions" a decade ago has at the dawn of the 2020's been eclipsed by the increased influence of malicious media manipulators and far-right antagonists. While a growing body of work addresses the techno-cultural dynamics at the root of this shift (Marwick \& Lewis, 2017; Phillips, 2018; Benkler, Faris, \& Roberts, 2018), the historical role of anonymous and pseudonymous online subcultures in this shift remains understudied. How exactly did these communities manage to dramatically (re)configure their often relatively apolitical subcultural practices towards political ends? What role did extremists, like those on $/ \mathrm{pol} /$, have in radicalising more politically ambivalent communities? To touch on these questions, this paper asks what the Tumblr-4chan raids can retroactively teach us about the political crystallisation of online subcultures halfway through the $2010 \mathrm{~s}$.

Historically, the paper scrutinises 2014 and 2015 as an alleged inflection point when Anglo-American online subcultures "got political" (Bernstein 2015; Beran 2019). Many accounts position Gamergate, a series of events leading to the harassment of feminist games journalists, as a pivotal turning point in the unification and far-right shift of various online subcultures (Phillips, 2018; Beran, 2019, p. 136-9). Without necessarily diminishing Gamergate's influence, this paper aims to present a more nuanced genealogical account of the alleged politicisation of online subcultures in the mid-2010s. It positions the 2014 Tumblr-4chan raids as an alternative microcosm that already featured many of the elements that would later attain greater visibility on a much larger scale, including the leaderless and "ironic" style of politics of the so-called "alt-right" in 2016 and 2017 (Heikkilä, 2017; Hawley, 2017) and the covert presence of white supremacists within seemingly tongue-in-cheek conflict (Marwick \& Lewis, 2017). 
This study first conducts a quantitative, data-driven analysis using datasets from the three spaces directly involved in the 2014 raids: Tumblr, 4chan/b/, and 4chan/pol/. Responding to the call by Phillips, Coleman, and Beyer to "accurately map the repeated, fractured, reconfiguring mobilizations emerging from anonymous and pseudoanonymous spaces" (2017), the paper maps the 2014 raids as a "born digital' sociocultural controversy" following Burgess and Matamoros-Fernández's study on Gamergate (2016). Drawing from the digital method of issue mapping (Rogers, 2013; Rogers, Sánchez-Querubín, \& Kil, 2015), the study engages in a "transcoding between milieus" (Deleuze \& Guattari, 1987) by visualising and interpreting networks of hyperlinks and cross-mentions between Tumblr, /b/, and /pol/. While some data-driven studies exist on these three spaces individually, this paper is methodologically unique in offering a "cross-subcultural microhistory". It will operationalise existing and contested popular-academic narratives on the antagonistic relationship between Tumblr and 4chan, notably by Nagle (2017) and Beran (2019). It will also be the first data-driven research into $4 \mathrm{chan} / \mathrm{b} /$ within this timeframe.

After mapping the event, the research then engages with the question: how can the raids be read as invoking political identification within the three milieus? Specifically, it concerns how discourse surrounding the raids can be seen to articulate political sensibilities. The paper does so by drawing from Mouffe's notion of "the political", which is concerned with "collective forms of identification" that deal "with the formation of 'us' as opposed to 'them"' (2013, p. 15), and such subjectivity is attained through "insertion in discursive/affective signifying practices, involving words, affects and actions" (2013, p. 71-3). In doing so, it aims to explain how politically divergent subcultures, whether they be progressive, reactionary, or seemingly nihilistic, can act co-constitutively, in turn challenging the notion of "subculture" as predominantly constructed by its opposition to a "mainstream" culture.

\section{References}

Benkler, Y., Faris, R., \& Roberts, H. (2018). Network Propaganda: Manipulation, Disinformation, and Radicalization in American Politics. Oxford: Oxford University Press.

Beran, D. (2019). It Came from Something Awful: How a Toxic Troll Army Accidentally Memed Donald Trump into Office. New York: All Points Books.

Bernstein, J. (2015). In 2015, The Dark Forces Of The Internet Became A Counterculture. Buzzfeed News.

https://www.buzzfeednews.com/article/josephbernstein/in-2015-the-dark-forces-of-theinternet-became-a-countercult\#.phAQpLDjy

Burgess, J., \& Matamoros-Fernández, A. (2016). Mapping Sociocultural Controversies across Digital Media Platforms: One Week of \#gamergate on Twitter, YouTube, and Tumblr. Communication Research and Practice, 2(1), 79-96.

Deleuze, G., \& Guattari, F. (1987). A Thousand Plateaus: Capitalism and Schizophrenia. Minnesota: University of Minnesota Press. 
Fairclough, R, Mulderrig, J, \& Wodak, R. (2006). Critical Discourse Analysis. In Discourse Studies: A Multidisciplinary Introduction, edited by Teun Van Dijk, 357-79. London: SAGE Publications Ltd.

Hawley, G. (2017). Making Sense of the Alt-Right. New York: Columbia University Press.

Heikkilä, N. (2017). Online Antagonism of the Alt-Right in the 2016 Election. European Journal of American Studies, 12(2).

Internet Historian. (2017). The Tumblr-4chan Wars. [Video]. YouTube. https://www.youtube.com/watch?v=SvjwXhCNZcU

Marwick, A., \& Lewis, R. (2017). Media Manipulation and Disinformation Online. [Report]. Data \& Society.

https://datasociety.net/pubs/oh/DataAndSociety_MediaManipulationAndDisinformationO nline.pdf

Miltner, K. M. (2014). "There's no place for lulz on LOLCats": The role of genre, gender, and group identity in the interpretation and enjoyment of an Internet meme. First Monday, 19(8).

Mouffe, C. (2018). For a Left Populism. London: Verso.

Nagle, A. (2017). Kill All Normies: The Online Culture Wars from Tumblr and 4chan to the Alt-Right and Trump. Winchester: Zero Books.

Nissenbaum, A., \& Shifman, L. (2017). Internet Memes as Contested Cultural Capital: The Case of 4chan's /b/ Board. New Media \& Society, 19(4), 483-501.

Phillips, W. (2015). This Is Why We Can't Have Nice Things: Mapping the Relationship between Online Trolling and Mainstream Culture. Cambridge: MIT Press.

Phillips, W. (2018). The Oxygen of Amplification: Better Practices for Reporting on Extremists, Antagonists, and Manipulators Online. [Report]. Data \& Society. https://datasociety.net/wpcontent/uploads/2018/05/1_PART_1_Oxygen_of_Amplification_DS.pdf

Phillips, W., Beyer, J., \& Coleman, G. (2017). Trolling Scholars Debunk the Idea That the Alt-Right's Shitposters Have Magic Powers. Motherboard. https://motherboard.vice.com/en_us/article/z4k549/trolling-scholars-debunk-the-ideathat-the-alt-rights-trolls-have-magic-powers

Rogers, R. (2013). Digital Methods. Cambridge: MIT Press.

Rogers, R., Sánchez-Querubín, N., \& Kil, A. (2015). Issue Mapping for an Ageing Europe. Amsterdam: Amsterdam University Press. 
shutdown4chan. (2014). \#ShutDown4chan [Tumblr post]. \#ShutDown4chan. https://shutdown4chan.tumblr.com/post/88296900118/its-time-to-shutdown-4chanorgjoin-us-on-july

\title{
DEEP VERNACULAR WEB TRANSCODING: A COMPARATIVE ANALYSIS OF THE HYPER-ANTAGONISTIC “KEKISTAN” MEME ON YOUTUBE AND 4CHAN IN 2017
}

\author{
Marc Tuters \\ University of Amsterdam
}

With Trump's inauguration, posters to the subcultural Web forum 4chan imagined themselves as victors in "The Great Meme War". In this period, the appearance of "fashy" (or fascist) memes within social media feeds was tied up in the rise of a new type of online ultra-nationalistic political movement referred to as the "alt-right". This paper offers a historical investigation of one such "alt-right" meme: Kekistan. In 2017, a contingent of Trump supporters - drawn together by a dark sense of humour - imagined themselves as the citizens of the fictional nation state of Kekistan at war with the forces of liberal political correctness. While having initially developed on 4chan, on YouTube, dozens of Kekistan-theme channels emerged, as for example in the genre of "protest LARPing" videos, in which Trump supporters would antagonize their opponents by flying the flag of Kekistan whose design was clearly based on that of the Nazi's Reichskriegsflagge. This paper will trace a narrative arc of how the use and meaning of this "meme" transformed over time and across platforms, as well as offering an historical perspective onto a cross section of user-generated "alt-right" Web culture from a period of time when the movement appeared ascendant. Using "kekistan" as a query to compile a historical corpus of 4chan posts and YouTube videos from this period - many of which the platform has since censored - the paper will present a snapshot of a relatively brief moment in time when a "deep vernacular Web" subculture associated with 4chan exploded into public view.

The paper conceptualizes this cross-platform phenomenon in terms of Deleuze and Guattari's concept of "transcoding between milieus" (1987 p. 322), according to which "[e]ach milieu has its own code", and transcoding scrutinises "the manner in which one milieu serves as the basis for another" (313). As opposed to assuming a one-way "dissemination" of a propagandistic message from 4chan as the source to YouTube as the target, this concept allows the paper to describe a conversation between the platforms. (This conversational aspect is, for example, demonstrated by the finding that YouTube is by far the most linked-to platform in the 4chan dataset and that many of the early Kekistan-themed videos within YouTube appear to summarise discussions that had taken place on 4chan.) Recounting this cross-platform narrative arc offers a framework for describing how the vernacular practices and technical affordances of 4chan constitute a local "code" that serves as the basis for a transcoding relationship between the platforms. The paper argues for this as a more useful concept than alternative and commonly used metaphors, whether drawn from the legacy of mass 
communications studies (propaganda), or else borrowed from evolutionary biology (memes) or epidemiology (viruses).

As extremism watchdogs organizations remarked at the time, Kekistan may be understood as a cultural artefact of the so-called "alt-right". Although never particularly coherent as a movement, in 2017 the term "alt-right" was used to refer to a broad network of actors drawn together in their mutual shared opposition to encroachments on "free speech", whether by campus officials, social media platforms, or simply "the establishment". Whilst sharing some of the same rhetorical techniques of populists, the "alt-right" can also be understood as a communication style that was marked by an unrelenting and extreme type of antagonism combined with a sophisticated use of vernacular Web culture. It was particularly associated with 4chan's "deep vernacular" subculture, in which speech and action are governed by peculiar axioms such as "Poe's Law", which obscures a speakers' intent behind the veils of "dissimulative identity play" (de Zeeuw \& Tuters, 2020). Together, these qualities led historians of American conservatism to refer to the "alt-right" as a "genuinely new phenomenon" (Hawley, 2017, p. 50).

Through Deleuze and Guattari's conceptual lens, this paper considers 4chan's "deep vernacular" subculture as a kind of template for the "alt-right's" supposed novelty. Whether in the form of memes, slang expressions, links to videos, or whole narrative constructs (such as in the case of "Kekistan"), individual 4chan posters use a technique of "nebulous othering" to negotiate a sense of belonging to an anonymous, ephemeral, and essentially chaotic community, through the practice of collectively mocking a vague enemy (Tuters \& Hagen, 2019). 4chan is often framed as "influencing" other parts of the Web, and nowhere is the idea of 4chan's influence greater than within 4chan itself. While such myths of influence have been studied in relation to earlier "hacktivist" and "trolling" subcultures emerging from 4chan's /b/ board, as the paper discusses, the myth of Kekistan was primarily the product of 4chan's "politically incorrect" discussion board, $/ \mathrm{pol} /$, which came to eclipse /b/ in popularity in the mid-2010's and which is commonly associated with the emergence of the "alt-right" (Hawley, 2017). Even though one cannot be sure about the identity of anonymous posters on 4chan, experts have long believed the sites' demographics to be "primarily white, male and somewhat privileged". While the problem of "toxic technoculture" has become increasingly recognized in the years since "Gamergate" with its well-known attacks on female games journalists and feminist media studies scholars, the issues of misogyny has arguably long been present in culture of webforums. As such, the analysis considers Kekistan as a transcoding of the a subculture typically associated with the "underground" milieu of forums and comment sections, to much more public and "mainstream" milieus - as, for example, well captured in the image, common in 2017 , of young men flying the flag of Kekistan in mocking counter-protests at anti-Trump demonstrations.

In dialogue with these types of characterizations of the artefacts of the "alt-right", through empirical analysis the paper asks: how did Kekistan mean different things at different points and in different milieus? In spite of its manifest absurdity, the paper argues that Kekistan is significant as a case study for how, for a brief period of time, a long-stand subcultural Web imaginary became enlisted in the service of a new mode of "hyper-antagonistic" political speech which opposed the pure people of the "deep 
vernacular Web" against a corrupt and gerontocratic elite, associated with the forces of "political correctness". The paper thus considers how a subcultural style with its roots in 4chan served to unify otherwise disparate actors (including trolls, white nationalists, and bored teenage boys) in a humorous type of protest that briefly served to gloss over real ideological differences between these actors - a coalition that would become increasingly untenable in the course of 2017. In the context of broader discussions concerning the strategic appropriation of Web subculture to advance a far-right agenda, the paper aims to communicate the relative nuance of these milieus and the transcoding between them, at a moment in time when the "alt-right" still appeared ascendant.

\section{References}

de Zeeuw, D, \& Tuters, M. (2020). Teh Internet Is Serious Business: on the Deep Vernacular Web and Its Discontents. Cultural Politics 16(2).

Deleuze, G, Guattari, F. (1987). A Thousand Plateaus. Minneapolis: University of Minnesota Press.

Hawley, G. (2017). Making Sense of the Alt-Right. New York: Columbia University Press.

Tuters, M., \& Hagen, S. (2019). (((They))) Rule: Memetic Antagonism and Nebulous Othering on 4chan. New Media \& Society, 1461444819888746.

\section{“KEEP PUSHING NEW SLANG": TRACING THE SPREAD OF EXTREME POLITICAL VERNACULAR FROM 4CHAN/POL/ TO BREITBART NEWS}

Stijn Peeters

University of Amsterdam

Tom Willaert

Vrije Universiteit Brussel

Fringe Internet platforms such as 4chan and other imageboards are often positioned as the "birthplace of memes" (Ludemann, 2018), or places where vernacular artefacts are produced that "cross over" to other platforms. Through their vernacular production, such fringe platforms can then have a profound impact on popular culture and politics despite their relative obscurity. In this paper, we empirically study whether the vernaculars of one of these spaces - the extremely vitriolic "politically incorrect" sub-forum of 4chan, /pol/ - indeed has meaningful external impact, in this case on the comment section of Breitbart, a right-wing news site that was found to have played a significant agendasetting role in 2016 U.S. presidential elections (Benkler, Faris, \& Roberts, 2018). We argue that a cross-over, or "transcoding", of /pol/'s vitriolic vernacular would mark an expansion of its hyper-antagonistic politics to the more mainstream Breitbart. 
There is some reason to suspect that a cross-over from /pol/ to elsewhere may be happening, as /pol/ but also other fringe platforms like it - e.g. Gab or parts of Reddit have become associated with an increasingly popular (far) right-wing political discourse. As an antisemitic and white supremacist space (Ludemann, 2018), /pol/ has also been associated with terrorist attacks in the United States, Canada, and New Zealand. While it is relatively obscure, $/ \mathrm{pol} /$ is an interesting object of study in that the phrases, images, and stereotypes initially developed there sometimes move beyond the confines of 4chan to find broader cultural uptake. The appropriation of "Pepe the Frog" is perhaps the most (in)famous recent example of this pattern, when in the mid 2010's it became an icon for the "alt-right". Other examples that have resonated beyond /pol/ itself are QAnon (the idea of an enigmatic figure claiming to be a White House insider; Hagen et al., 2019), Pizzagate (a crackpot conspiracy theory; Tuters et al., 2018), as well as much of the vocabulary associated with the "manosphere" (Marwick \& Caplan, 2018).

While Pepe is arguably the most famous example of /pol/'s cultural (re-)production, it is far from the only one. From antagonistic slang ("cuck", "redpilling", "normies") to endless variations on the classic antisemitic "Happy Merchant" caricature, /pol/ is a heavily vernacular space characterised by rapid linguistic and cultural innovation. What these innovations generally have in common is that they reflect a vitriolic far-right ideology, based on a "nebulous other" that is pitted against a mostly white, male, traditionalist identity (Tuters \& Hagen, 2019). The platform's cultural production then is largely concerned with antagonistically articulating this other through the use of caricatures, slurs, and conspiracy theories.

The extreme nature of the linguistic innovations on /pol/ is especially of concern when considering their potential to take root elsewhere. At the same time, one cannot however assume that their original valence will remain intact, since processes of translation are at work, as shown by the recent appropriation of Pepe as a symbol of the civil protests in Hong Kong, which seemed devoid of "alt-right" connotations. With this in mind, less ambiguous artefacts, for example vitriolic phrases and racial slurs, may be more likely to remain their "original" valence.

This paper empirically studies the circulation of far-right linguistic artefacts between platforms. We compare two large datasets of comments posted on /pol/ and Breitbart News using methods borrowed from computational linguistics. Like /pol/, Breitbart rose to prominence in the context of the 2016 presidential elections in the United States. It has been described as a harbinger of the "alt-right" and a place where conspiracy theories are cultivated (Davis, 2019). It thus presents a place where ideas and vernacular from $/ \mathrm{pol} /$ may resonate. Our central research question therefore is whether we can find evidence of /pol/'s extreme vernacular spreading to Breitbart, and whether this constitutes the spread of /pol/'s politics.

We investigate this by tracing the occurrences of vernacular typical for $/ \mathrm{pol} /$ on Breitbart. If /pol/'s culture does indeed resonate on Breitbart, one would expect to see phrases or words that are popular on $/ \mathrm{pol} /$ to also appear and grow in popularity there, in a similar context. Our dataset comprises 20 months of comments, centred around the 2016 U.S. elections. If we do find evidence of 4chan's vernacular spreading, this could constitute what is popularly referred to as "widening the Overton window", i.e. the practice of 
making hitherto taboo topics fair game for discussion. As such, the discourse on Breitbart - a site with a far larger reach than /pol/ - could then be characterised as becoming more extreme.

We extract relevant case studies from these large datasets by first extracting words that occur relatively often in either dataset. We are especially interested in "neologisms" here, i.e. words that do not occur in standard dictionaries (in this case, the Google ngrams corpus), marking vernacular and linguistic innovation. A significant majority of these neologisms that first appear within our data are found to occur on 4chan before they occur on Breitbart, indicating that /pol/ indeed functions as an incubator of vernacular that then circulates to other platforms.

Next, we qualitatively zoom in on a small number of case studies from these neologisms that occur on /pol/ first. Here we are especially interested in words that clearly "spike" in popularity in Breitbart after they have first become popular on 4chan. Examples of these include "borealis", a white supremacist phrase referring to Northern Europe; "Kalergi", a reference to the racist "Great Replacement" conspiracy theory; and "Iugenpresse", a classic fascist term referring to supposedly untruthful mainstream media.

These case studies, in which we qualitatively explore the context in which the terms are used as well as around which events they were used particularly often, complement the larger quantitative data analysis. Together, they show that indeed the circulation of /pol/produced phrases and words extends to other platforms; and that, even though the "milieu" they are adopted in is different from /pol/, many of these phrases remain unambiguously hateful, antagonistic, or otherwise "extreme". This indicates that /pol/s reputation as an incubator of extreme politics is warranted, and that the imageboard could potentially have a significant impact on discourse beyond 4chan itself.

\section{References}

Benkler, Y., Faris, R., \& Roberts, H. (2018). Network Propaganda: Manipulation, Disinformation, and Radicalization in American Politics. Oxford: Oxford University Press.

Davis, M. (2019). A new, online culture war? The communication world of Breitbart.com. Communication Research and Practice, 5(3), 241-254.

Hagen, S., De Zeeuw, D., Peeters, S., Jokubauskaitè, E., \& Briones, Á. (2019). Understanding Normiefication: A Cross-Platform Analysis of the QAnon Conspiracy Theory. Digital Methods Initiative Wiki.

https://wiki.digitalmethods.net/Dmi/WinterSchool2019Normiefication

Ludemann, D. (2018). /pol/emics: Ambiguity, scales, and digital discourse on 4chan. Discourse, Context \& Media(24), 92-98.

Marwick, A. E., \& Caplan, R. (2018). Drinking male tears: Language, the manosphere, and networked harassment. Feminist Media Studies, 18(4), 543-559. 
Tuters, M., \& Hagen, S. (2019). (((They))) rule: Memetic antagonism and nebulous othering on 4chan. New Media \& Society, 1461444819888746.

Tuters, M., Jokubauskaitè, E., \& Bach, D. (2018). Post-Truth Protest: How 4chan Cooked Up the Pizzagate Bullshit. M/C Journal, 21(3).

\title{
ALT-TECH, ALT-MEDIA, ALT-RIGHT: FAR-RIGHT MILIEUS AND ALTERNATIVE MEDIA DIETS AFTER THE 2019 "SUMMER OF DEPLATFORMING"
}

\author{
Emillie de Keulenaar \\ Simon Fraser University \\ Kings College London \\ Jack Wilson \\ University of Warwick
}

Historically, Facebook, Twitter, and YouTube have used the argument of affording their users "free speech" as both a selling point as well as a means to distinguish themselves as being democratic, "participatory" alternatives to mass media (Langlois, 2013). In the past half-decade, these platforms have however taken on a more proactive role of moderating their user base and content following myriad controversies - including harassment campaigns (Jeong, 2019), the fake news-mediated dissemination of conspiracist narratives (Venturini et al., 2018), and inter-ethnic violence up to and including genocide (Mozur, 2018). Furthermore, this has manifested in the implementation of anti-hate speech measures intended to prevent incitement to hatred or violence towards specific nationalities, sexualities, genders, or ethnic and religious groups (Gordon, 2017). Indeed, mainstream platforms now have many strategies to contain hate speech, from demoting content in recommendations and search results (Constine, 2019) to redirecting users to educational material in an effort to "deradicalize" them (The Redirect Method, 2016).

Thus far, the primary method in the attempt to "sanitise" content has been "deplatforming". Deplatforming is the banishment of users and deletion of their content for hate speech and related offenses. This approach has embedded these platforms in ongoing battles of ideas as to what constitutes an offence to racial, gender, and other identities, how to protect such identities from discrimination, and where to delimit the boundaries of free speech. This practice has sparked fierce resistance from the deplatformed users themselves - particularly with those who identify with political positions on the right. Particularly in the Anglo-American context, these aggrieved users (or former users) often assert that these platforms operate as part of a conspiracy concerned with censoring ideas rather than offensive speech (Rogers, forthcoming).

Media and scholarly research on deplatforming has mainly studied the effectiveness of such measures, and - considering that figures like the Breitbart columnist Milo Yiannopolous or the influential conspiracy theorist Alex Jones "mellowed" their hitherto 
"extreme" political language and that their audience has thinned (Rogers, forthcoming) such studies have given reason to believe that deplatforming is an effective strategy for policing hate speech and protecting users from radicalisation (Chandrasekharan et al., 2017). However, studies on the effectiveness (and ethics) of deplatforming have yet to engage with deplatforming's effects beyond this initial migration to alternative platforms. Although it is unlikely that alternative platform ecologies will constitute a real alternative to mainstream platforms (Roose, 2017), this paper poses two claims: that they nonetheless constitute a substantial alternative media diet for users in mainstream social media platforms, and that, as such, they work not in isolation but in tandem with mainstream platforms as co-dependent "milieus" (Deleuze \& Guattari, 1987, p. 313).

That these alt-tech milieus emerge at all suggests a certain robustness and ideological momentum on the part of these hate-driven communities, even if their overall numbers have diminished. The ongoing presence of such milieus is not addressed in scholarship that has focused - to date - on deplatformed individuals (Rogers, forthcoming). We hypothesise that while deplatforming may initially diminish the size of a deplatformed milieu, those that remain may be - or may become - more engaged, and in this sense deplatforming may actually work to reconstitute and cohere these communities. While the former is a well-known claim (Ohlheiser, 2016), we further hypothesise that deplatforming can also energise a circulation of the deplatformed content across various milieus. This post-deplatforming circulation can occur as a "migration" to alternative platforms, as evidenced by a re-appearance of several banned neo-Nazi YouTube videos on Bitchute, archive.org, and other alternative media websites (OILab, 2019). Further, removed content may be the subject of reconstitution, or "replatforming", on the platform it was initially banned from. Both allow the removed content to be consumed and exchanged on mainstream platforms again, whether as hosted by the platform itself or in the form of external URLs and other exchanged objects.

To engage with these hypotheses, we propose a two-phased study drawing from digital methods (Rogers 2013). Firstly, we map the migration of deplatformed YouTube videos after its 2019 "summer of deplatforming", when the video service deleted thousands of red-flagged channels and videos (OILab, 2019). Can we see the deplatformed videos move to alternative milieus, and if so, where do they appear? As a basis for this is a corpus of metadata, including video titles, of thousands of far-right YouTube videos gathered in late 2018. We first map which videos and channels are still hosted on YouTube today by requesting their present status through the YouTube API. Then, for the deleted videos, we query their metadata 1 ) on search engines to extract domains and map the breadth of alternative websites, 2) on Bitchute and alternative media websites to inquire their prevalence on alternative platform ecologies, and 3) on the archival websites archive.org and archive.is to investigate possible survival as archived content. The second stage entails identifying whether the surviving content eventually re-appears or "replatforms" on mainstream websites. We use the base corpus of deplatformed YouTube metadata, as well as derivative "natively digital objects" (Rogers 2013) as found on alternative platforms (such as alternative video titles or archive.org URLs), and query this back on YouTube, as well as in Reddit posts and Tweets. By identifying which videos re-appeared, the aim is to arrive at a fine-grained mapping of deplatformed content by not only tracing where they "migrate" but also if and where they "replatform". 


\section{References}

Chandrasekharan, E., Pavalanathan, U., Srinivasan, A., Glynn, A., Eisenstein, J., \& Gilbert, E. (2017). You Can't Stay Here: The Efficacy of Reddit's 2015 Ban Examined Through Hate Speech. Proceedings of the ACM on Human-Computer Interaction, 1(CSCW), 1-22.

Constine, J. (2019). Instagram now demotes vaguely 'inappropriate' content. TechCrunch. http://social.techcrunch.com/2019/04/10/instagram-borderline/

Deleuze, G., \& Guattari, F. (1987). A Thousand Plateaus: Capitalism and Schizophrenia. Minnesota: University of Minnesota Press.

Gordon, G. S. (2017). Atrocity Speech Law: Foundation, Fragmentation, Fruition. Oxford: Oxford University Press.

Hagen, S., Burton, A., Wilson, J., \& Tuters, M. (2019). Infinity's Abyss: An Overview of 8chan. OILab.eu. https://oilab.eu/infinitys-abyss-an-overview-of-8chan/

Jeong, S. (2019). Zoë Quinn. The New York Times.

https://www.nytimes.com/interactive/2019/08/15/opinion/gamergate-zoe-quinn.html

Langlois, G. (2013). Participatory Culture and the New Governance of Communication: The Paradox of Participatory Media. Television \& New Media, 14(2), 91-105.

Mozur, P. (2018). A Genocide Incited on Twitter, With Posts from Myanmar's Military. The New York Times. https://www.nytimes.com/2018/10/15/technology/myanmarfacebook-genocide.html

Ohlheiser, A. (2016). Banned from Twitter? This site promises you can say whatever you want. Washington Post. https://www.washingtonpost.com/news/theintersect/wp/2016/11/29/banned-from-twitter-this-site-promises-you-can-say-whateveryou-want/

OlLab. (2019). 4chan's YouTube: A Fringe Perspective on YouTube's Great Purge of 2019. OlLab.eu. https://oilab.eu/4chans-youtube-a-fringe-perspective-on-youtubesgreat-purge-of-2019/

Rogers, R. (2013). Digital Methods. Cambridge: MIT Press.

Rogers, R. (forthcoming). Deplatforming: Following extremist internet celebrities to Telegram and alternative social media. European Journal of Communication.

Roose, K. (2017). The Alt-Right Created a Parallel Internet. It's an Unholy Mess. The New York Times. https://www.nytimes.com/2017/12/11/technology/alt-right-internet.html

The Daily Beast. (2017). Daily Stormer Site Moves to Dark Web After Shutdown. The Daily Beast. https://www.thedailybeast.com/daily-stormer-site-moves-to-dark-web-after- 
shutdown

The Redirect Method. (2016). The Redirect Method. http://redirectmethod.org

Venturini, T., Bounegru, L., Gray, J., \& Mauri, M. (2018). A Field Guide to 'Fake News' and Other Information Disorders. SSRN. http://fakenews.publicdatalab.org/ 